\title{
Experimental and numerical investigation of controlled disturbances development from two sources in supersonic boundary layer
}

\author{
G. L. Kolosov*, A. D. Kosinov, A. N. Semenov and A. A. Yatskikh
}

* Correspondence: kolosov@itam. nsc.ru

Khristianovich Institute of

Theoretical and Applied Mechanics SB RAS, Novosibirsk 630090, Russia

\begin{abstract}
The paper presents the experimental results and the results of direct numerical simulation of the development and interaction of two wave trains from two point sources of controlled disturbances in a supersonic boundary layer on a flat plate with an incident flow Mach number of 2.5. Sources were located parallel to the leading edge of the model. For the introduction of controlled disturbances into the boundary layer, the normal component of the mass flow rate was varied in the calculations. In the experiment, periodic glow discharges at a frequency of $20 \mathrm{kHz}$ were used. In both cases, the disturbances sources worked synchronously. Mass flow rate pulsations were measured and recorded in sections, parallel to the leading edge of the model, near the maximum of disturbances along the boundary layer. In the experiment, a constanttemperature hot-wire anemometer was used. After performing a discrete Fourier transform, the spatial distributions of disturbances, the beta-spectra were determined, and the wave characteristics of the development of disturbances downstream were estimated. In addition, direct numerical simulation of the downstream development of disturbances from a single source was performed. The work presents a comparison of experimental and theoretical calculated data. The paper discusses the effects inherent in the interaction of unstable traveling controlled disturbances from two sources operating synchronously.
\end{abstract}

Keywords: DNS, Computational modeling, Supersonic boundary layer, Compressibility, Discharge, Flat plate, Hot-wire anemometer, Instability, Turbulence

\section{Introduction}

Laminar-turbulent transition in the boundary layers is one of the fundamental problems in fluid dynamics, which has great practical significance. It is considered that flow turbulization in the boundary layer occurs due to the appearance and evolution of various types of disturbances, their growth and non-linear interaction with each other with the formation of vortex structures localized in space and time (wave packets, turbulent spots, streamwise streaks, soliton-like coherent structures etc.) [1-6]. To predict the laminar-turbulent transition, it is necessary to understand the dominant processes and their mechanisms responsible for the breakdown of the laminar regime. We note here such nonlinear mechanisms of

(C) The Author(s). 2019 Open Access This article is distributed under the terms of the Creative Commons Attribution 4.0 International License (http://creativecommons.org/licenses/by/4.0/), which permits unrestricted use, distribution, and reproduction in any medium, provided you give appropriate credit to the original author(s) and the source, provide a link to the Creative Commons license, and indicate if changes were made. 
interaction of disturbances as an oblique transition and subharmonic resonance $[7,8]$. These scenarios are possible both in the subsonic case and in the supersonic one. In a subsonic boundary layer, an oblique transition is initiated by a nonlinear interaction of two oblique waves of finite amplitude, symmetrical with respect to the flow direction. The effectiveness of such a path of turbulization is shown by the results of computational and experimental studies of the laminar turbulent transition in the channel and in the boundary layer [9-12]. Subharmonic resonance implies the interaction of three traveling waves: the main Tollmien-Schlichting wave and a pair of subharmonic waves [7, 13-15]. In the final stage of the transition, on longitudinal structures secondary unsteady disturbances (secondary high-frequency instability) develop, that eventually leads to the establishment of a turbulent regime.

One of the most informative methods for the experimental study of laminarturbulent transition in boundary layers is the initiation of artificial pulsations. Controlled input of artificial disturbances with given parameters allows one to study their development and compare them with the results of the theory and calculations. Today, for the case of supersonic flow velocities in the boundary layer, single localized sources are most often used to excite controlled disturbances. Controlled disturbances from them have a wide wave spectrum, which allows to study the development of disturbances with different angles of inclination. Such an approach is effective in studying linear and weakly nonlinear evolution of disturbances both in the boundary layer on a flat plate and on a swept wing $[16,17]$.

Earlier, in studies at low subsonic flow speeds with the help of several sources, attempts were made to simulate the interaction of disturbances, as well as to control the laminar-turbulent transition process. In [18], in the boundary layer of a flat plate, Tollmien-Schlichting waves were introduced from a vibrating ribbon and from a number of holes or a slit on the model surface. It was found that, depending on the phase relation between the introduced disturbances, it is possible to control the position of the transition point from laminar to turbulent flow. In addition, the method of generating disturbances from several localized sources is effective in studying the mechanisms of interaction of disturbances. Thus, in [19], it was found that stationary waves are amplified in the separation zone on a flat plate model and high-frequency pulsations are excited when controlled disturbances are introduced through two slits on the model surface.

For the case of supersonic flow velocities, as in the subsonic case, the study of laminar-turbulent transition using controlled disturbances from several sources seems promising. Such an approach was used in [20], where in the boundary layer of a cone, using a set of localized sources of controlled disturbances, instability waves were generated as the most growing waves according to the linear theory. However, in this work, measurements were carried out only for different values of the longitudinal coordinate and the wave spectrum of the disturbances was not determined. For the development of the method of generation of controlled disturbances by several localized sources in the boundary layers at supersonic flow velocities, detailed studies are needed. The purpose of this work is to experimentally and numerically simulate the development and interaction of traveling unstable disturbances from two localized sources in a supersonic boundary layer on a flat plate at Mach number 2.5. 


\section{Problem statement, numerical method and experimental setup}

During the calculations, the motion of gas is described by the known Navier-Stokes, continuity, energy and state equations. In this calculations $c_{p}=1006.43 \mathrm{~J} / \mathrm{kg} \cdot \mathrm{K}\left(c_{p}-\right.$ specific heat at a constant pressure) and thermal conductivity was taken in according to the kinetic theory. The temperature of the main flow was set equal to $T_{r}=128.8 \mathrm{~K}$. Mach number was taken $\mathrm{M}=2.5$, pressure was equal to $4800 \mathrm{~Pa}$ and it corresponded to unit Reynolds number $\operatorname{Re}_{1}=(\mathrm{U} \rho / \mu)_{\infty}=8 \cdot 10^{6} / \mathrm{m}$.

The computational domain is schematically presented in Fig. 1.A'BCD' is the plate with the disturbance sources. The following coordinate system was used in the calculations and experiment: the $x$-axis is aligned with the incoming flow, the $z$-axis is parallel to the leading edge of the model, and the $y$-axis is normal to the plate surface. The origin of the coordinate system was located on the leading edge, and the value $z=0 \mathrm{~mm}$ corresponds to the symmetry line of the model (see Fig. 1). Sources of controlled disturbances were holes with a diameter of $1 \mathrm{~mm}$ on the surface of the model. In the calculations, we considered two cases of the location of the sources. The first case is a single source of disturbances located at $z=0 \mathrm{~mm}$, the second case is two sources of disturbances that are $6 \mathrm{~mm}$ apart from each other and located at $z= \pm 3 \mathrm{~mm}$. In both cases, the sources were installed at a distance of $30 \mathrm{~mm}$ from the leading edge of the model. Note, that Fig. 1 shows the second case with two sources of disturbances. Length of a plate equaled $140 \mathrm{~mm}$, before a plate the area of $5 \mathrm{~mm}$ was set. Conditions of an adiabatic wall were realized on a plate. Height of the computational domain approximately corresponded to about $20 \mathrm{~mm}$, and on the upper border (EFGH) nonreflecting boundary conditions were laid down. Width of the domain was set equal to $40 \mathrm{~mm}$, and it was enough that controlled disturbances from the source extinguished on lateral borders. Moreover, on the sides ABHE and DCGF there are non-reflecting boundary

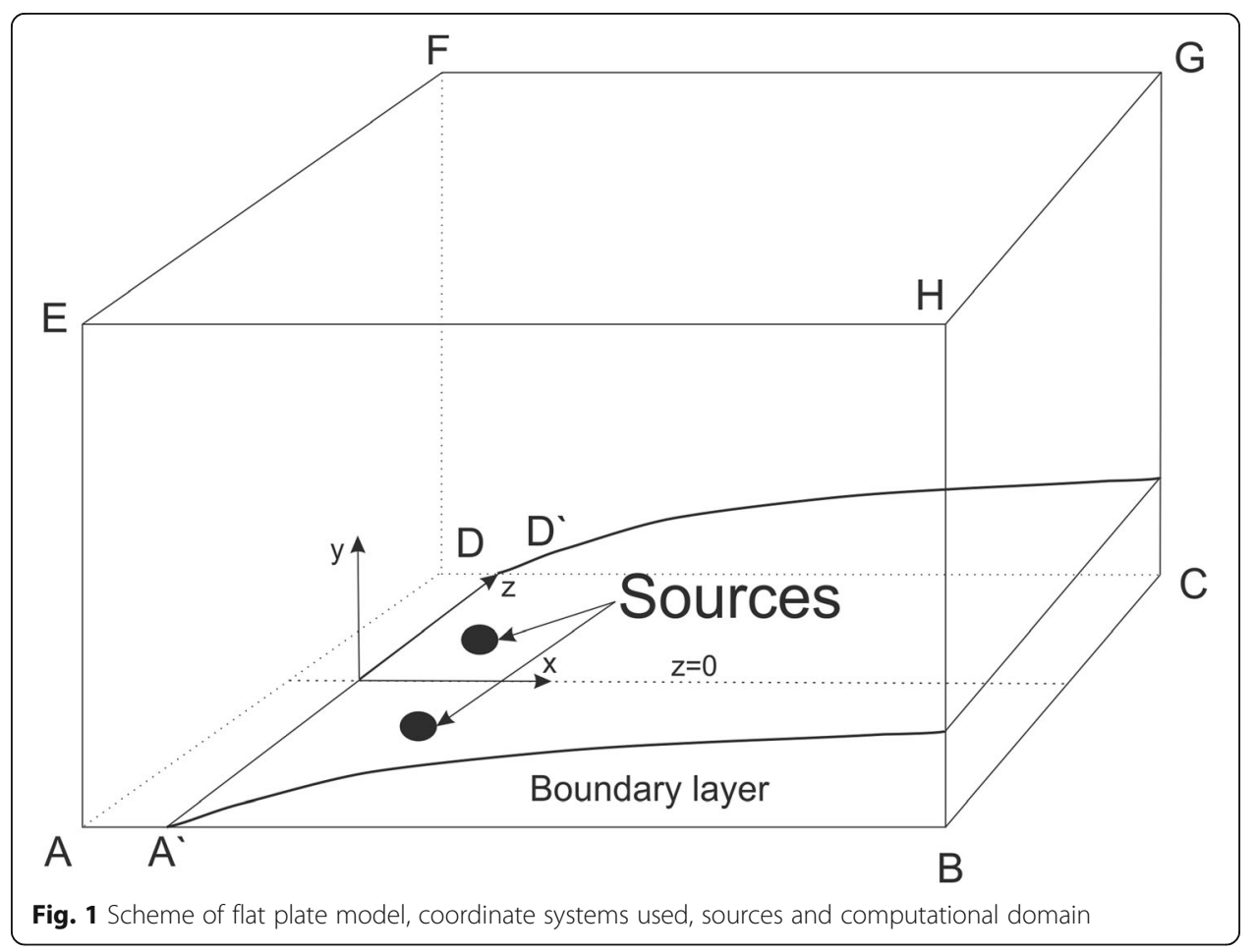


conditions, which means that the fluctuations on the walls were set equal to zero during the calculation. In this case, the side walls were installed at such a distance along the $z$-axis, so that they did not affect the disturbances excitation and development downstream. Conditions of the oncoming flow were laid down on border ADFE. Exit conditions were set on the border BCGH.

In this work, the following structured grid was chosen: the quantity of cells on $x$-coordinate was equal to 1200 , on $z-200$ and on $y-400$. Also, the condensation at the surface of the model on $y$-coordinate was used. For the solution of the task the program complex ANSYS was used [21]. To solve the three-dimensional Navier-Stokes equations in the framework of this problem, we used a density-based solver and an implicit scheme of MUSCL of the third order. The splitting of convective flows was made with using the AUSM method. The problem was solved in two stages. At the first stage, the stationary problem was solved. At the second stage, the task was solved in the presence of a periodic disturbances, which were created by the air injection through a holes. Disturbances were a normal component of the mass flow, whose amplitude was distributed in time by law $|\sin (2 \pi f t)|$, where $f=10 \mathrm{kHz}$. It means that disturbances at a frequency of $20 \mathrm{kHz}$ from each source of disturbances are introduced into the boundary layer. Note that this article discusses the synchronous operation of two sources. This means that the phase shift was chosen to be zero. Duration of calculation equaled 1000 microseconds, and the time step was equal to $10^{-2} \mu \mathrm{s}$.

Experiments were performed in a T-325 long-duration blowdown low-noise supersonic wind tunnel, at the Institute of Theoretical and Applied Mechanics, Siberian Branch of the Russian Academy of Sciences, at Mach number $M=2.5$ and unit Reynolds number $\operatorname{Re}_{1}=(8 \pm 0.1) \cdot 10^{6} \mathrm{~m}^{-1}$. A steel flat plate with a sharp leading edge and a couple of localized sources of controlled disturbances were used as an experimental model. The plate had the following dimensions: width $-200 \mathrm{~mm}$, length $-370 \mathrm{~mm}$, thickness $-10 \mathrm{~mm}$. The leading edge thickness did not exceed $0.1 \mathrm{~mm}$. The model was fixed in the central plane of the test section at approximately a zero angle of attack with an alignment error of $0.06^{\circ}$.

According to the calculations, two sources of controlled disturbances were located at a distance of $30 \mathrm{~mm}$ from the leading edge of the model at values of the transverse coordinate $z= \pm 3 \mathrm{~mm}$. The design of each source is similar to those used in the works $[16,17]$. A discharge chamber located inside the model and connected with the boundary layer through a hole of $0.4 \mathrm{~mm}$ diameter was used. An electrode inserted was installed inside the camera and was isolated from the model. The discharge was ignited when the sufficient voltage between the electrode and the model is achieved.

To excite controlled disturbances inside the sources, a glow discharge was ignited at a frequency of approximately $20 \mathrm{kHz}$. The ignition circuit is shown in Fig. 2. It consists of a high voltage source, ballasts for limiting the discharge current and two highvoltage high-speed switches. Control of switches is performed using a two-channel pulse generator. The generator creates pulses with a duration of approximately $10 \mu \mathrm{s}$ with a repetition rate of $20,004.6 \mathrm{~Hz}$. The generator channels, as in the calculations with two sources, were synchronized (phase shift was set to $0^{\circ}$ ). Oscillograms of control signals are presented in Fig. 3. The corresponding oscillograms of voltage drop at the sources of controlled disturbances during the experiment are also shown in Fig. 3. When the signal comes to the switch, it opens and the parasitic capacitance of the 


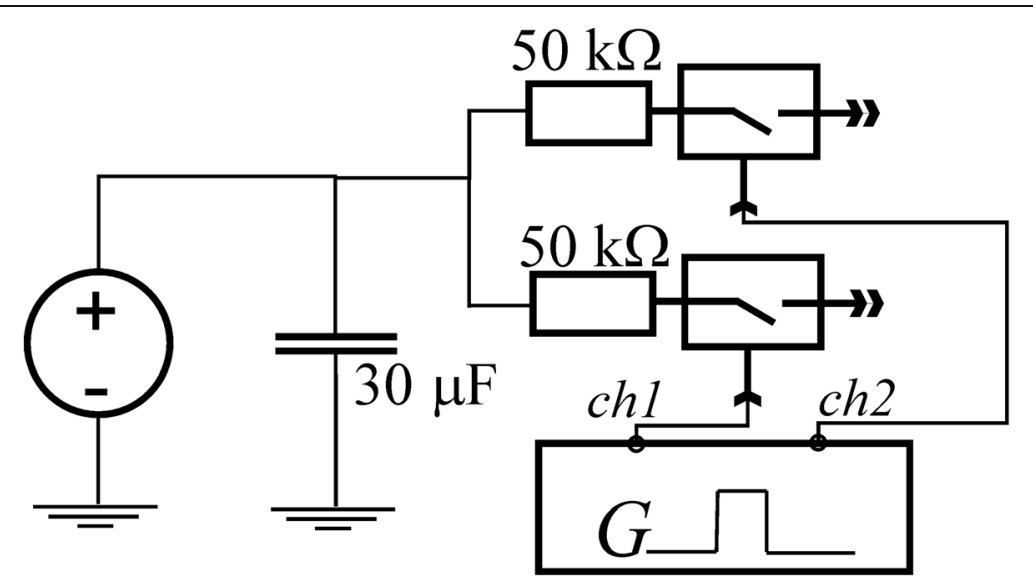

Fig. 2 The ignition circuit

source of controlled disturbances starts charging. When the breakdown voltage is reached, a glow discharge is ignited, during burning of which the voltage drop across the electrodes remains constant. After $10 \mu \mathrm{s}$, the switch receives a zero signal and it closes, and the discharge is interrupted.

Pulsations in a supersonic flow were measured using constant-temperature hot-wire anemometer (CTA). A tungsten wire of $10 \mu \mathrm{m}$ in diameter and $1.7 \mathrm{~mm}$ in length was used. The overheat rate of the hot-wire probe was set to $0.7-0.8$, and it means that the measured disturbances up to 95\% consisted of mass flow fluctuations $\left(m^{\prime}\right)$ [22-24]. The dc output voltage $(E)$ of the anemometer was measured with an Agilent 34401A digital voltmeter. The anemometer pulse signal $\left(e^{\prime}\right)$ was digitalized with a 12-bit analogue-digital convertor (ADC). The ADC sampling frequency was $750 \mathrm{kHz}$. At each measurement point, 8 signal realizations of 65,536 points were recorded synchronously with the discharge ignition. Non-dimensional mass flux pulsations were calculated using the following formula:

$$
m^{\prime}(t)=\frac{(\rho U)^{\prime}}{\overline{\rho U}} \approx \frac{e l(t)}{S_{\rho U} \cdot E},
$$

where $\overline{\rho U}$ is the local mass flux, $S_{\rho U} \approx 0.25 \pm 0.01$ is the hot-wire probe sensitivity for mass flow pulsations. The stagnation temperature during the experiments was in the range $283-288 \mathrm{~K}$. Therefore, the variation of the overheat ratio of the hot-wire probe was insignificant.
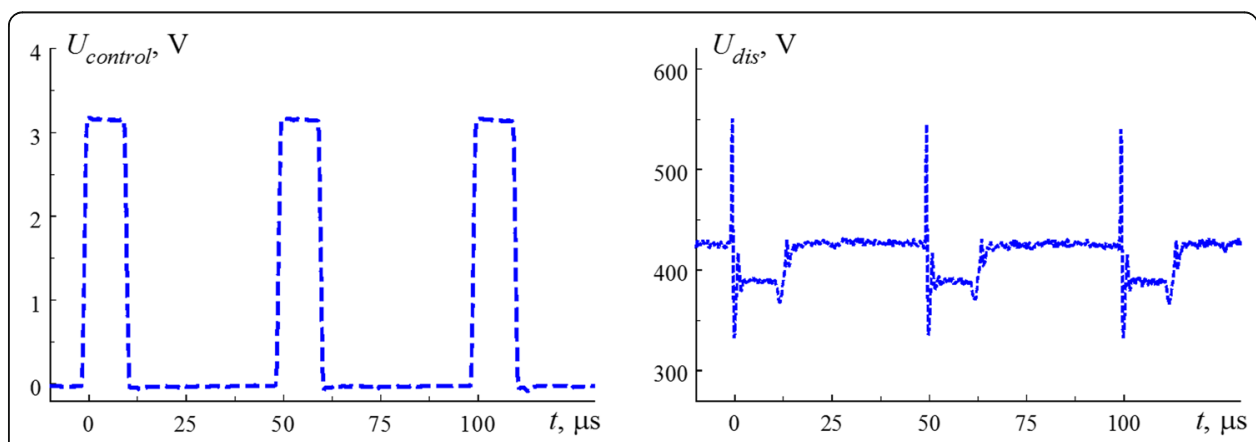

Fig. 3 Oscillograms of control signals (left) and voltage drop (right) at the sources during the experiment 
Hot-wire probe was installed in the supersonic part of the boundary layer in the region of the maximum level of disturbances. This allows us to use the calibration of the hot-wire anemometer obtained in supersonic flow. In this layer mean mass flow is about $\overline{\rho U} \approx 0.7(\rho U)_{\infty}$. A trajectory of the probe was parallel to the leading edge at a constant distance to the plate surface. Cross sections was measured at $x=80,100 \mathrm{~mm}$ from the leading edge. The probe was moved in the $z$-directions with the help of traversing gears with which the wind tunnel was equipped. The measurement accuracy was about $0.1 \mathrm{~mm}$. The distance between nearby measurement points in space of the hot-wire probe was about $0.5 \mathrm{~mm}$.

The spectral analysis was conducted to obtain the detailed information about the evolution of the controlled disturbances:

$$
m_{f \beta}^{\prime}=\frac{\sqrt{2}}{T \cdot \delta} \sum_{j, k} m^{\prime}\left(t_{k}, z_{j}\right) e^{-i \cdot\left(\beta z-2 \pi f t_{k}\right)} \Delta z_{j} \Delta t
$$

where $T$ - record length, $\delta=1 \mathrm{~mm}$ - characteristic thickness of the boundary layer. The amplitude and phase distributions were defined as:

$$
\begin{aligned}
& A_{f \beta}=\left|m_{f \beta}^{\prime}\right|, \\
& \Phi_{f \beta}=\operatorname{arctg}\left[\frac{-\operatorname{Im}\left(m_{f \beta}^{\prime}\right)}{\operatorname{Re}\left(m_{f \beta}^{\prime}\right)}\right] .
\end{aligned}
$$

\section{Results and discussions}

We emphasize once again that in this work the main calculations were performed for the case when two sources of disturbances worked synchronously. In addition, calculations were carried out with a single source of disturbances located along the center line of the plate symmetry ( $x=30 \mathrm{~mm}$ from the leading edge of the model, $z=0 \mathrm{~mm}$ ). This had to be done in order to reveal the distinctive features of the interaction of disturbances from two sources.

Figure 4 shows the isolines of total disturbances (for all frequencies) in sections $x=$ $60,80,100 \mathrm{~mm}$ from the leading edge of the model in cases of a single source and in the case of synchronous operation of two sources. Here is the ratio of the instantaneous amplitudes of the mass flow rate pulsations to the average mass flow rate locally. Solid red lines - positive amplitudes, dotted blue - negative. The step between the lines is $0.001 \%$, the maximum value of the amplitudes of the isolines in absolute value is $0.011 \%$. In isolines, there is a positive and negative defect of the mean flow. An expansion of the wave trains and its spreading towards positive and negative values of the transverse $z$-coordinate is observed. In the case of a single source of disturbances, the formation of side "petals" is observed downstream, having a slope in the coordinates $(t$, $z$ ). In the case of two sources of disturbances, "petals" are formed in the region $|z|>3$ $\mathrm{mm}$, in the place where there is no influence from the neighboring source of disturbances. In the central part of the computational domain (near $z=0 \mathrm{~mm}$ ), one can see the disturbances interaction downstream. In this case, it is a sequential alternation of positive and negative defects of mass flow. Note that in the case of synchronous operation of sources, the pattern of isolines is symmetric about the center line $z=0 \mathrm{~mm}$. 


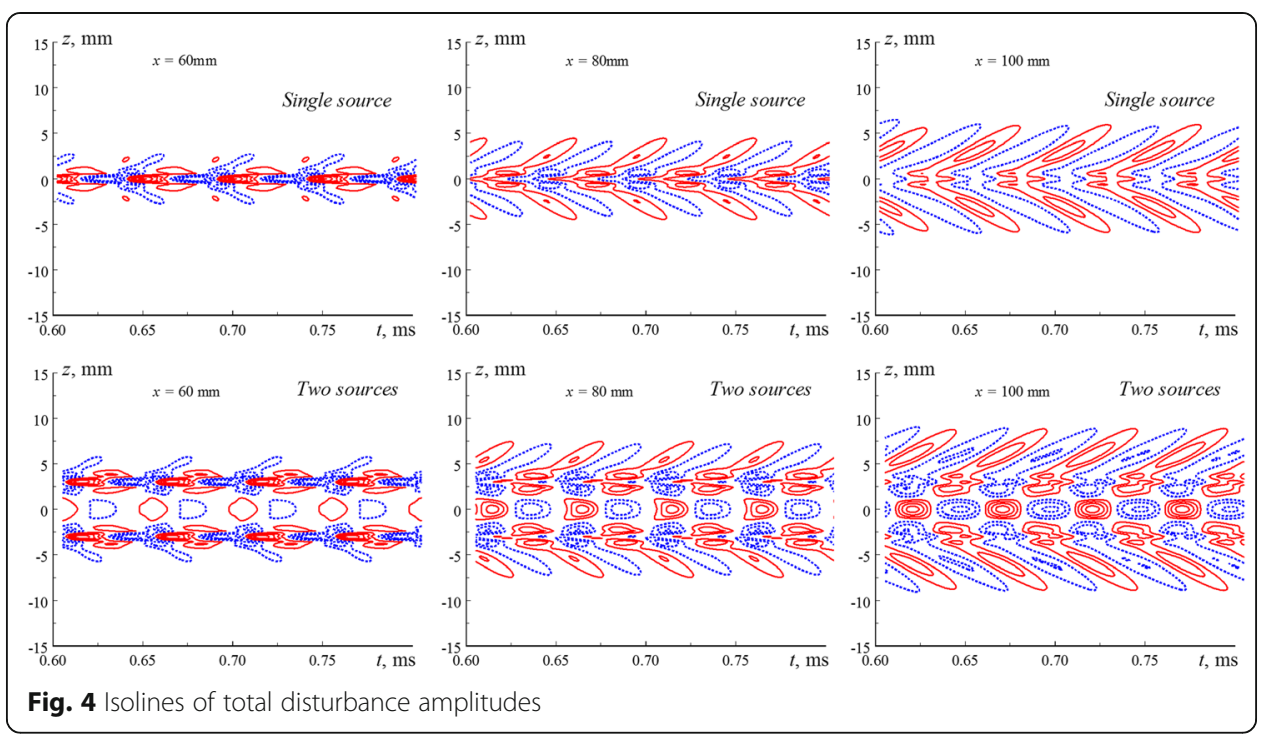

Further, according to formula (1), the Fourier transform in time was performed for the main frequency $f=20 \mathrm{kHz}$. Figure 5 shows the development of amplitudes of controlled disturbances downstream for the case of a single source, and for the case of two sources of disturbances operating synchronously. In the case of a single disturbance source, computational results are in accordance with the experimental results in a supersonic boundary layer on a flat plate under controlled conditions [16]. Thus, two symmetric main peaks are observed, with positive and negative values of the $z$-coordinate, whose maxima downstream are displaced from the center line $z=0 \mathrm{~mm}$. The shift is explained by the fact that in the supersonic boundary layer the most energy-carrying traveling disturbances are inclined to the incident flow [16]. Also, an increase in the amplitudes of the wave trains and its spreading are observed. In the case of two sources of disturbances working synchronously, we note here a more complex form of interacting wave trains. However, again we can note the observed expansion of the wave trains downstream and the growth of disturbance amplitudes. In this case, an increase in the amplitude of disturbances at $|z|>5 \mathrm{~mm}$ is similar to the case of a wave train development from a single source [16]. In the region $|z|<5 \mathrm{~mm}$, the presence of several peaks of disturbance amplitudes is the result of the interaction of wave trains from two sources. At the same time, with synchronous operation of the sources, there is a
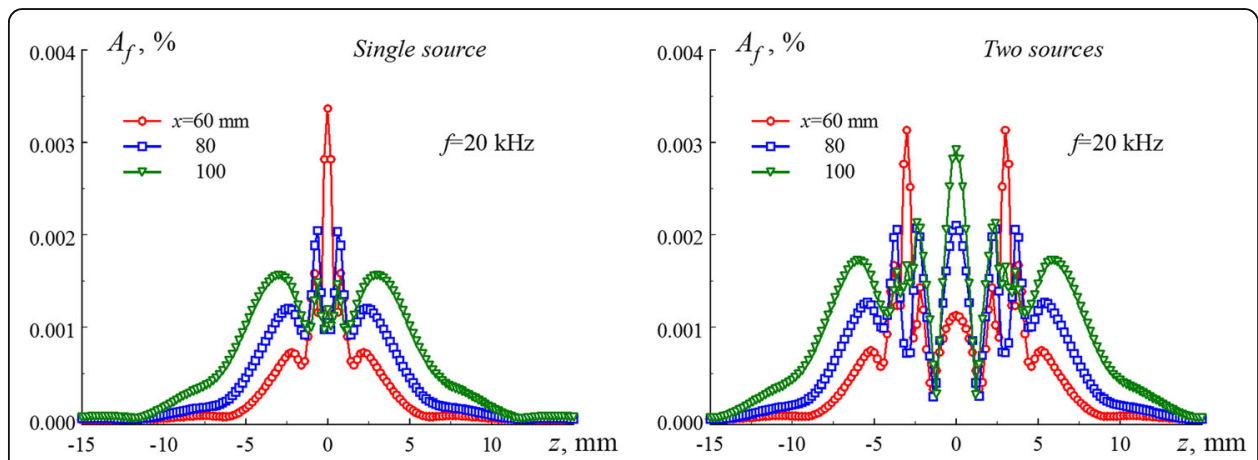

Fig. 5 Amplitude distributions of disturbances in the spanwise direction at $f=20 \mathrm{kHz}$ 
noticeable increase in disturbances at $z=0 \mathrm{~mm}$, since the summation of synchronous waves is taking place in this region.

A more visual picture is obtained after the discrete Fourier transform in space (see formula (1)) and considering the $\beta$-spectra of the development of disturbances downstream (Fig. 6). In the case of a single discharge, there are two symmetric maximum perturbations located at $\beta= \pm 1.08 \mathrm{rad} / \mathrm{mm}$, which grow downstream. This is again consistent with experiments on a flat plate under controlled conditions [16]. Another picture in the $\beta$-spectra is observed for two sources of disturbances operating synchronously. Thus, again there is an increase in the amplitude of disturbances, but we can see the presence of several maxima in the $\beta$-spectra, as well as several nodes where the amplitude of the disturbances is almost zero, namely at $\beta= \pm 0.52,1.56,2.6$, $3.68 \mathrm{rad} / \mathrm{mm}$. In a simplified form, this effect can be explained as follows: in the boundary layer, the disturbances at the point $(x, z)$ are the sum of monochromatic waves from two sources $\sum_{i} A_{i} \sin \left(2 \pi f t+\beta_{i}\left(z-z_{0}\right)+\alpha_{r} x\right)+\sum_{i} A_{i} \sin \left(2 \pi f t+\beta_{i}\left(z+z_{0}\right)+\alpha_{r} x\right)$, where the amplitude $A_{i}\left(\beta_{i}\right)$ is determined after the Fourier transform (formula (1)) and where the summation is performed over all transverse wave numbers $\beta_{i}, z_{0}$ - source positions, $z_{0}=3 \mathrm{~mm}$. According to the known formulas, the sum of sines can be reduced to the form: $2 \sum_{i} A_{i} \sin \left(2 \pi f t+\beta_{i} z+\alpha_{r} x\right) \cos \left(\beta_{i} z_{0}\right)$. Under the sign of summation, the expression $\sin \left(2 \pi f t+\beta_{i} z+\alpha_{r} x\right)$ defines the spectrum from a single source. $2 \cos \left(\beta_{i} z_{0}\right)$ determines the modulation of the spectrum of a single source upon transition to the disturbance spectrum from two sources operating synchronously. So, it is possible to determine the nodes in the beta spectrum, based on the expression: $\beta_{i}^{0}=\frac{\pi}{2 z_{0}}+\frac{\pi n}{z_{0}}$ where $n$ is an integer. This expression gives the values $\pm 0.52,1.57,2.61,3.66 \mathrm{rad} / \mathrm{mm}$, which is in accordance with the values obtained in the calculation. Also, the presence of a factor of 2 explains the observed doubling of the amplitude of the main peak.

Next, other wave characteristics of wave trains development from a single source and two sources operating synchronously were obtained. Fig. 7 presents the dispersion dependence $\alpha_{r}(\beta)$ and amplification rates $\alpha_{i}(\beta)$. In the case of two sources of disturbances, the domain of definition of these characteristics is somewhat narrower than in the case of a single source, since the region of the most unstable disturbances near the main peak $\beta=1 \mathrm{rad} / \mathrm{mm}$ in the $\beta$-spectrum is also narrower due to the interaction of disturbances. However, despite the interaction that has taken place, and the amplitude of

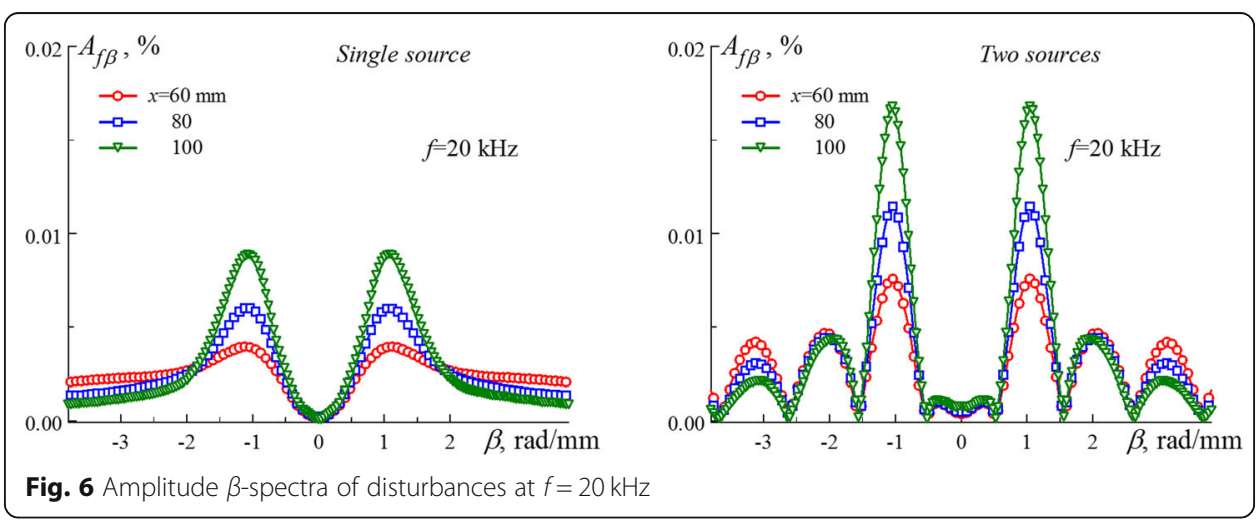


disturbances has doubled, the wave characteristics remain the same compared to the development of disturbances from a single source. In this case, for the most unstable disturbances at $\beta= \pm 1.08 \mathrm{rad} / \mathrm{mm}$, the angle of inclination of the wave to the incident flow was $\pm 71^{\circ}$, which is in accordance with previously performed experiments under controlled conditions in the supersonic boundary layer on a flat plate [16].

Thus, summing up the results of direct numerical simulation, it can be said that in the case of a single source of disturbances, the obtained wave characteristics of the development of unstable disturbances are in accordance with previously performed experiments in a supersonic boundary layer on a flat plate in controlled conditions. In the case of two sources of disturbances operating synchronously, the calculations revealed the effect of the interaction of two wave trains, which resulted in the formation of a characteristic interference pattern in the $\beta$-spectrum with a set of several nodes and antinodes.

The experiments also took place in conditions when the sources of disturbances worked synchronously. In this case, two sections were measured at a distance of $x=80$ and $100 \mathrm{~mm}$ from the leading edge. The measurements were performed in the supersonic part of the boundary layer in the region of the maximum level of pulsations. The anemometer probe moved parallel to the leading edge of the model. Here it is also necessary to give some information on the natural unstable wave (with other frequencies) of the supersonic flat plate boundary layer. Amplitude-frequency spectra of the total signal at a distance of $x=80 \mathrm{~mm}$ in two different transversal positions are shown in Fig. 8. The spectrum on the left represents data beyond the limits of the wave trains $(z=-18 \mathrm{~mm})$, i.e. for natural pulsations; that on the right corresponds to the region of interaction of wave trains from two sources of controlled disturbances $(z=-0.5 \mathrm{~mm})$. In both spectra of total pulsations, disturbances at frequencies of $40,60,80 \mathrm{kHz}$ are distinguished. At the same time, these disturbances have a constant amplitude at all measured points when the hot-wire probe moves parallel to the leading edge of the model. We assume that the presence of these peaks in the spectrum is associated with electromagnetic interference, which is generated with each ignition of the discharge, i.e. with a base frequency of $20 \mathrm{kHz}$. In turn, as seen from Fig. 8 (right), the sources of controlled disturbances introduced pulsations at frequency $f=20 \mathrm{kHz}$ in the supersonic boundary layer. The results for the development and interaction of controlled disturbances at frequency $20 \mathrm{kHz}$ are presented below. In addition, comparing the amplitude spectra presented in Fig. 8, we can conclude that the influence of the sources of the controlled

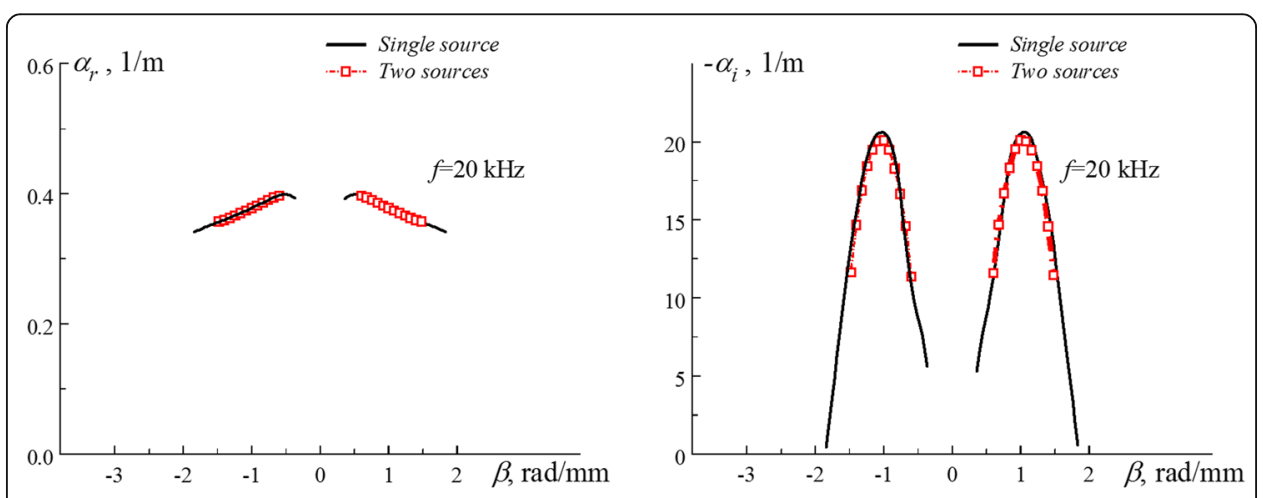

Fig. 7 Dispersion relations $a_{r}(\beta)$ (left) and amplification rates (right) for disturbances at $f=20 \mathrm{kHz}$ 
disturbances is negligible and does not change the structure and spectral distribution of natural disturbances.

Figure 9 shows the amplitude distributions downstream for disturbances at the source operating frequency of $20 \mathrm{kHz}$. Similar to the results of direct numerical simulation, in the central region $|z|<5 \mathrm{~mm}$ there are several peaks in the amplitude distributions of disturbances - the result of the interaction of wave trains from two sources. In contrast to the calculation data, the experiment does not show an increase in the disturbance amplitude downstream. This may be due to the fact that in measuring sections $x=80$ and $100 \mathrm{~mm}$, the hot-wire probe was located at different values of the average mass flow rate, i.e. at different values $y / \delta$.

Amplitude spectra of disturbances for transverse wave numbers $\beta$ for $x=80$ and 100 $\mathrm{mm}$ are presented in Fig. 10. For visual comparison, Fig. 10 shows both experimental data and data obtained using DNS. In experiments, controlled perturbations are distinguished in the range of wave numbers $\beta=-2.5-2.5 \mathrm{rad} / \mathrm{mm}$. Outside this range, the amplitude of the controlled disturbances is much less than the natural pulsations of the boundary layer. We also note that the amplitude of controlled disturbances in direct numerical simulation is much less than in experimental studies. Nevertheless, there is a qualitative agreement between experimental and calculated data. In the experiments, there are several maxima in the beta spectrum, as well as several nodes, where the amplitude of the disturbances is small. The values of wave numbers $\beta$ of nodes and antinodes in experiment and calculations are close.

It should also be noted that in the experiment there is a significant peak in the amplitude wave spectra near the values of the wave numbers $\beta=0 \mathrm{rad} / \mathrm{mm}$. This peak does not correspond to the linear theory of stability and, most likely, does not relate to the detected effect of wave trains interference from two sources of disturbances in the supersonic boundary layer of a flat plate. In addition, this peak is not related to the mechanism of subharmonic resonance, since in the wave spectra at a frequency of 10 $\mathrm{kHz}$ (as at frequencies of $30,50 \mathrm{kHz}$ ) disturbances are not distinguished for all values of $\beta$. This peak at $\beta=0 \mathrm{rad} / \mathrm{mm}$ is most likely associated with the electromagnetic interference from discharges discussed earlier. This is also confirmed by the fact that in the region $\beta=0 \mathrm{rad} / \mathrm{mm}$ the same peaks with the same amplitude of about $0.1 \%$ were found in the wave spectra at frequencies of 40 and $60 \mathrm{kHz}$, whereas at $\beta>0.4 \mathrm{rad} / \mathrm{mm}$ the disturbances don't stand out.
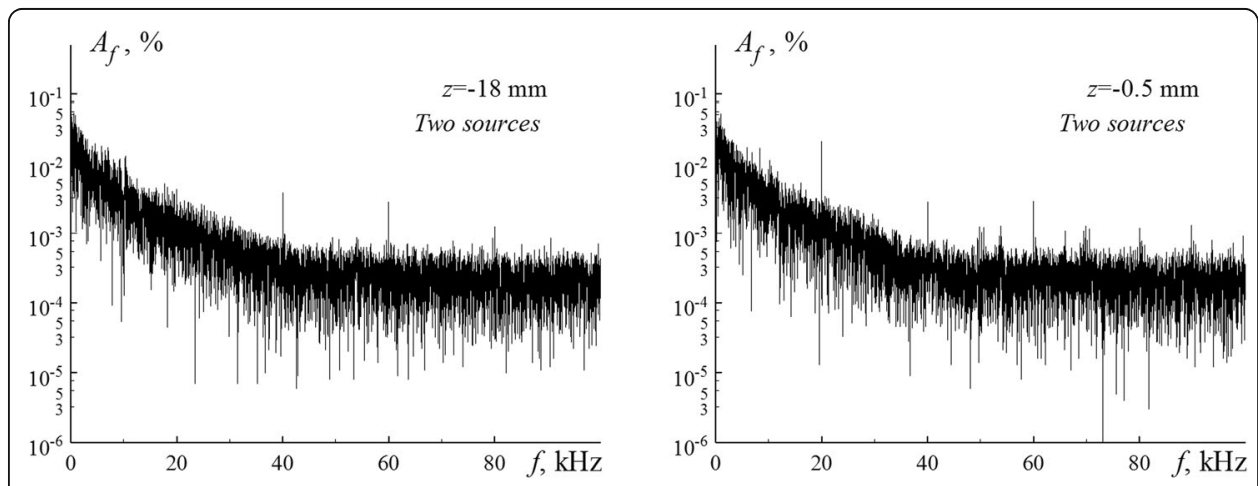

Fig. 8 Amplitude spectra of the total pulsations at $x=80 \mathrm{~mm}$ and $z=-18 \mathrm{~mm}$ (left), $z=-0.5 \mathrm{~mm}$ (right) 


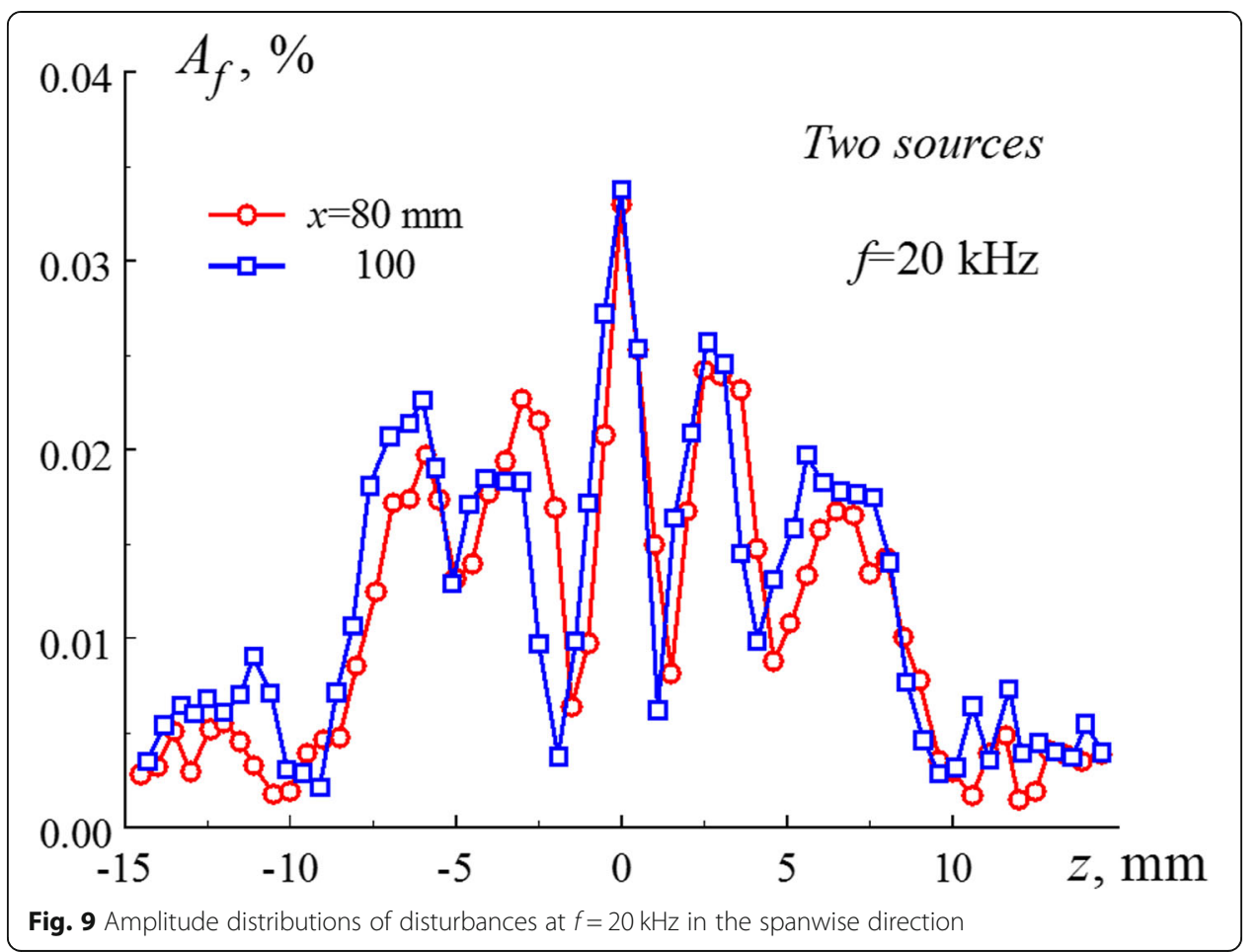

\section{Conclusions}

A numerical and experimental study of the development and interaction of controlled disturbances from two point sources in a supersonic boundary layer on a flat plate with a Mach number 2.5 has been carried out. In the case of a single source of pulsations, the wave characteristics of the development of unstable disturbances obtained in calculations are in accordance with previously performed experiments in a supersonic boundary layer on a flat plate. In the case of two sources of disturbances operating synchronously, the calculations revealed the effect of the interaction of two wave trains, which resulted in the formation of a characteristic interference pattern in the $\beta$-spectra with a set of several nodes and antinodes. Experimental results revealed the same effect and are in accordance with the calculations in the framework of direct numerical simulation. The work must be continued with the sources of disturbances working not

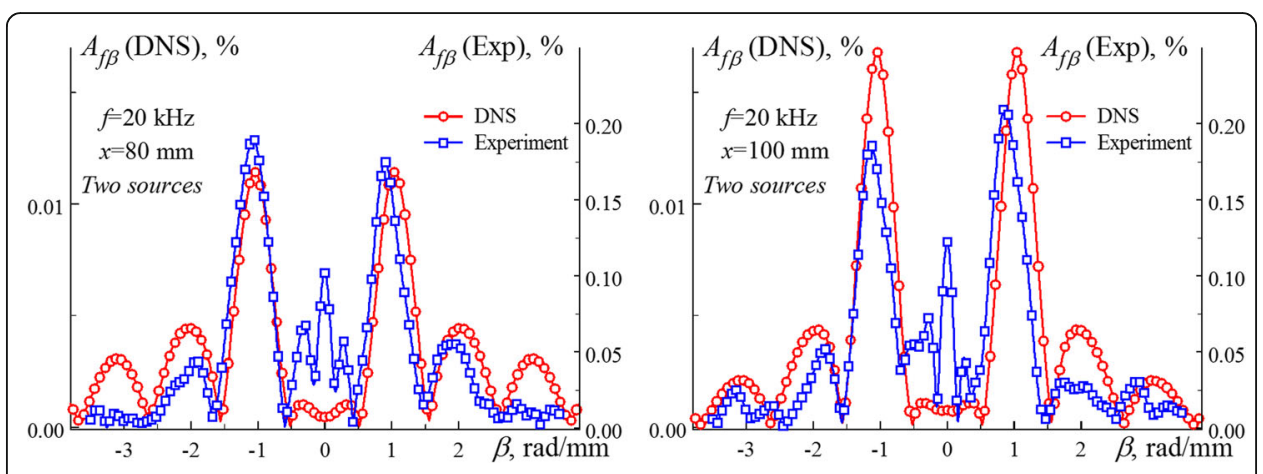

Fig. 10 Amplitude $\beta$-spectra of disturbances at $f=20 \mathrm{kHz}$ 
synchronously, but with a phase shift. In addition, it would be important to consider the effects of nonlinear interaction of disturbances, such as subharmonic resonance, when pulsations from independent sources are introduced into the supersonic boundary layer at different frequencies.

\section{Acknowledgements}

The authors would like to thank Afanasyev L. for designing a generator for synchronous operation of sources of disturbances, Semenov N.V., Ermolaev Yu.G. for assistance in conducting experiments, and Professor Kozlov V.V. for a discussion of the results on the interaction of controlled disturbances in the subsonic boundary layer.

\section{Authors' contributions}

ADK is responsible for the formulation of the problem. ANS carried out the numerical computation. GLK and AAY are responsible for preparing and conducting experiments, data processing. All authors participated in writing the article, read and approved the final manuscript.

\section{Funding}

The research was supported by RFBR (Grant No. 18-31-00171 mol_a).

The research was partly carried out within the framework of the Program of Fundamental Scientific Research of the state academies of sciences in 2013-2020 (project No. AAAA-A17-117030610125-7).

The work was performed using the equipment of the CCU "Mechanics".

\section{Availability of data and materials}

The datasets used and/or analyzed during the current study are available from the corresponding author on reasonable request.

\section{Competing interests}

The authors declare that they have no competing interests.

Received: 29 March 2019 Accepted: 6 June 2019

Published online: 05 July 2019

\section{References}

1. Boiko AV, Grek GR, Dovgal AV, Kozlov W (2002) The origin of turbulence in near-wall flows. Springer-Verlag, Berlin

2. Bippes H (1999) Basic experiments on transition in three-dimensional boundary layers dominated by crossflow instability. Prog Aerosp Sci 35:363-412

3. Grek G, Kozlov W, Ramasanov MP (1985) Three types of disturbances from the point source in the boundary layer. In: Kozlov W (ed) Laminar-turbulent transition: symposium, Novosibirsk, USSR July 9-13, 1984. Springer-Verlag, Berlin, p 267. https://doi.org/10.1007/978-3-642-82462-3 34

4. Kachanov YS (1994) Physical mechanisms of laminar-boundary-layer transition. Annu Rev Fluid Mech 26:411-482. https://doi.org/10.1146/annurev.fl.26.010194.002211

5. Lee CB (1998) New features of CS solitons and the formation of vortices. Phys Lett A 247:397-402. https://doi.org/10. 1016/S0375-9601(98)00582-9

6. Lee CB, Wu JZ (2008) Transition in wall-bounded flows. Appl Mech Rev 61:030802. https://doi.org/10.1115/1.2909605

7. Kachanov YS, Levchenko WY (1984) The resonant interaction of disturbances at laminar-turbulent transition in boundary layer. J Fluid Mech 138:209-247. https://doi.org/10.1017/S0022112084000100

8. Mayer CSJ, Wernz S, Fasel HF (2011) Numerical investigation of the nonlinear transition regime in a Mach 2 boundary layer. J Fluid Mech 668:113-149. https://doi.org/10.1017/S0022112010004556

9. Schmid PJ, Henningson DS (1992) A new mechanism for rapid transition involving a pair of oblique waves. Phys Fluids A 4:1986-1989. https://doi.org/10.1063/1.858367

10. Elofsson PA, Alfredsson PH (1998) An experimental study of oblique transition in plane Poiseuille flow. J Fluid Mech 358 177-202. https://doi.org/10.1017/S0022112097008288

11. Berlin S, Lundbladh A, Henningson D (1994) Spatial simulations of oblique transition in a boundary layer. Phys Fluids 6 : 1949-1951. https://doi.org/10.1063/1.868200

12. Chang CL, Malik MR (1994) Oblique-mode breakdown and secondary instability in supersonic boundary layers. J Fluid Mech 273:323-360. https://doi.org/10.1017/S0022112094001965

13. Craik ADD (1971) Non-linear resonant instability in boundary layers. J Fluid Mech 50:393-413. https://doi.org/10.1017/ S0022112071002635

14. Ermolaev YG, Kosinov AD, Semionov NV (1996) Experimental investigation of laminar-turbulent transition process in supersonic boundary layer using controlled disturbances. In: Duck PW, Hall P (eds) Nonlinear instability and transition in three-dimensional boundary layers. IUTAM symposium, Manchester, July 1995. Kluwer Academic Publishers, Dordrecht, Boston, London, p 17

15. Fezer A, Kloker M (2000) Spatial direct numerical simulation of transition phenomena in supersonic flat-plate boundary layers. In: Fasel HF, Saric WS (eds) Laminar-turbulent transition. IUTAM symposium, Sedona, September 1999. SpringerVerlag, Berlin, p 415

16. Kosinov AD, Maslov AA, Shevelkov SG (1990) Experiments on the stability of supersonic laminar boundary layers. J Fluid Mech 219:621-633. https://doi.org/10.1017/S0022112090003111

17. Kosinov AD, Kolosov GL, Semionov NV, Yermolaev YG (2016) Linear development of controlled disturbances in the supersonic boundary layer on a swept wing at Mach 2. Phys Fluids 28:064101. https://doi.org/10.1063/1.4952999

18. Ablaev AR, Grek GR, Dovgal AV, Katasonov MM, Kozlov W (2000) Transition to turbulence in a separation bubble caused by interaction of two oblique waves. Thermophys Aeromech 7:353-360 
19. Gilev VM, Kozlov W (1986) Influence of periodic blow-suction on the transition process in the boundary layer. Uche Zap TsAGI 3:27-33 in Russian

20. Corke TC, Cavalieri DA, Matlis E (2002) Boundary-layer instability on sharp cone at Mach 3.5 with controlled input. AIAA J 40:1015-1018. https://doi.org/10.2514/2.1744

21. ANSYS Fluent Theory Guide. Release 12.1 (2009) ANSYS, Inc

22. Yatskikh AA, Kosinov AD, Semionov NV, Smorodsky BV, Ermolaev YG, Kolosov GL (2018) Investigation of laminarturbulent transition of supersonic boundary layer by scanning constant temperature hot-wire anemometer. AIP Conf Proc 2027:040041. https://doi.org/10.1063/1.5065315

23. Smits AJ, Hayakava MKC (1983) Constant temperature hot-wire anemometer practice in supersonic flows. Exp Fluids 1:83-92

24. Kosinov AD, Semionov NV, Yermolaev YG (1999) Disturbances in test section of T-325 supersonic wind tunnel. Preprint 6-99, ITAM SB RAS, Novosibirsk

\section{Publisher's Note}

Springer Nature remains neutral with regard to jurisdictional claims in published maps and institutional affiliations.

Submit your manuscript to a SpringerOpen ${ }^{\circ}$ journal and benefit from:

- Convenient online submission

- Rigorous peer review

- Open access: articles freely available online

- High visibility within the field

- Retaining the copyright to your article

Submit your next manuscript at $\boldsymbol{\nabla}$ springeropen.com 